\title{
De la qualité entre partenaires responsables!
}

Ce n'est un secret pour personne; les acteurs du domaine de la politique de la santé ne sont actuellement pas engagés dans une culture de partenariat. Ce partenariat n'existe pas. Il n'est ni recherché ni promu par les autorités politiques, et les acteurs peinent à aller au-delà des clichés qui circulent au sein de chaque camp. La FMH ne tient évidemment pas à soutenir cette orientation.

Le politique ne maîtrise pas tout, et pour tenter de sortir de ce qui pour lui est une impasse, il s'est tourné de manière préférentielle vers les managers. La stratégie du management est donc, actuellement, prédominante dans le champ de la santé. Il n'est pas question ici de la rejeter en bloc: il s'agit plus d'en pointer l'idéologie univoque appliquée au domaine de la santé et du social, et c'est ce que j'ai tenté de faire dans l'article qui paraît dans ce numéro, et qui développe les idées esquissées dans cet éditorial.

Il m'est apparu intéressant de relever dans cette réflexion que la stratégie de la «bonne pratique managériale» se fonde sur un discours subtil qui s'articule autour des termes d'autocontrôle, d'autocorrection et surtout d'auto-évaluation. L'apothéose en est l'accréditation.

Cette évaluation se veut au service d'une démarche de qualité qui est aujourd'hui fixée légalement par le triptyque de l'économicité, de l'efficacité et de l'adéquation (art. 32 de la LAMal). Le tout est emballé et vendu dans un discours de responsabilité que le politique confond encore trop souvent avec la bonne volonté*.

Ce fétiche nommé «qualité» vise à fédérer la collectivité et les professionnels de la santé dans une sorte d'illusion groupale. Le discours sur la qualité est ici utilisé de manière perverse puisqu'on ne peut bientôt plus critiquer l'idéologie de ce concept sans être suspecté d'hérésie contre le bien public. La difficulté tient peut-être au fait que le moteur du discours sur la qualité n'est pas tant un défaut de qualité qu'il s'agirait urgemment de corriger mais bien le fantasme de la maîtrise: maîtrise des différences, des singularités et peut-être même des contestations sociales latentes qui, elles, coûtent très cher. Il est évident que si nous étions tous pareils et si nous nous comportions tous de la même manière, «comme le maître le voudrait», ce serait bien plus facile! Dans cette nouvelle illusion groupale, il n'y a donc pas de place pour une culture de la gestion des différences

Cela dit, face à ces blocages, la FMH et ses organisations de base ne restent pas passives puisqu'elles cherchent à élaborer, avec différents partenaires du champ de la santé, de nouveaux concepts incluant le sujet pour définir et gérer la qualité en médecine. Ce partenariat est cependant encore très balbutiant et fragile, en raison des a priori tenaces et du manque de soutien politique.

Pour conclure, il me semble que la relation entre partenaires ne pourra s'exercer que si chaque acteur peut se faire reconnaître comme responsable de ses dires et des conséquences de ceux-ci. Ça n'est qu'à cette condition que le politique, les assureurs, les médecins et les usagers pourront être reconnus comme des partenaires dans l'ensemble des négociations qui portent sur le champ de la santé. Et dans ces négociations aucun thème n'est a priori tabou pour la FMH.

R. Raggenbass, membre du Comité central de la FMH 


\section{Qualität zwischen verantwortungsbewussten Partnern!}

* Unter Verantwortung verstehe ich die Fähigkeit des Subjekts, zu seinen Worten und Taten zu stehen.
Es ist ein offenes Geheimnis: Die gesundheitspolitischen Akteure pflegen zurzeit keine Kultur der Partnerschaft. Eine solche Partnerschaft gibt es im Augenblick überhaupt nicht. Sie wird von der Politik weder angestrebt noch gefördert, und die Partner haben Mühe, die im jeweiligen Lager vorherrschenden Klischees zu überwinden. Die FMH will natürlich eine solche Ausrichtung nicht unterstützen.

Die Politik beherrscht nicht alles, und um aus dieser in ihren Augen als Sackgasse zu betrachtenden Situation herauszukommen, wendet sie sich vorzugsweise an die Manager. Im Gesundheitswesen dominiert nämlich heute die Managementstrategie. Diese soll aber hier nicht rundweg abgelehnt werden, sondern es geht viel eher darum, auf deren eindeutige, auf das Gesundheits- und Sozialwesen angewandte Ideologie hinzuweisen. Ich habe versucht, dies in dem in dieser Ausgabe erscheinenden Artikel darzulegen und die in diesem Editorial skizzierten Ideen näher auszuführen.

Ich hielt es für interessant, bei diesen Überlegungen hervorzuheben, dass die Strategie der «guten Managementpraxis» sich auf einen subtilen Diskurs rund um die Begriffe Selbstkontrolle, Selbstkorrektur und vor allem Selbstevaluierung stützt. Die Krönung dieses Prozesses ist dann die Akkreditierung.

Die Evaluierung will der Qualitätssicherung dienen, die heute mit dem Dreigestirn Wirtschaftlichkeit, Wirksamkeit und Zweckmässigkeit gesetzlich vorgeschrieben ist (Art. $32 \mathrm{KVG}$ ). All dies wird in einen Diskurs über Verantwortung* verpackt und verkauft, der von der Politik noch zu oft mit gutem Willen verwechselt wird.

Dieser Fetisch namens «Qualität» soll die Allgemeinheit und die Gesundheitsfachleute in einer Art Kollektivwahn zusammenführen. Der
Qualitätsdiskurs wird auf pervertierte Weise geführt, weil man die Ideologie hinter diesem Konzept bald nicht mehr kritisieren kann, ohne des Verrats am Gemeingut verdächtigt zu werden. Die Schwierigkeit liegt vielleicht darin, dass die treibende Kraft hinter dem Qualitätsdiskurs nicht so sehr ein dringend zu behebender Qualitätsmangel, sondern das Trugbild der Beherrschung ist: Beherrschung der Unterschiede, der Einzigartigkeit und vielleicht sogar der sehr teuren latenten sozialen Proteste. Eines ist klar: Wenn wir alle gleich wären und uns alle gleich verhielten, «wie der Herr es will», wäre es viel einfacher! Bei dieser neuen Kollektivillusion ist also kein Platz für eine Kultur des Umgangs mit Unterschieden.

Die FMH und ihre Basisorganisationen sind jedoch angesichts der verfahrenen Situation nicht untätig geblieben und versuchen, mit verschiedenen Partnern des Gesundheitswesens neue Konzepte zu erarbeiten, die das Subjekt einbeziehen, um die Qualität in der Medizin zu definieren und $\mathrm{zu}$ sichern. Diese Partnerschaft steckt jedoch erst in den Anfängen und ist wegen der hartnäckigen Vorurteile und der mangelnden politischen Unterstützung noch anfällig.

Abschliessend scheint mir, dass die Beziehung zwischen Partnern nur gepflegt werden kann, wenn jeder Beteiligte als ein Akteur anerkannt wird, der Verantwortung für seine Worte und deren Folgen übernimmt. Nur dann können Politik, Versicherer, Ärzte und Patienten als Partner in den gesamten Verhandlungen im Gesundheitswesen anerkannt werden. Und bei diesen Verhandlungen ist für die FMH kein Thema tabu.

R. Raggenbass, Mitglied des Zentralvorstandes der FMH 